of the pancakes. It is believed that the pancakes (kinks) mainly determine properties like flux pinning, flux flow and creep. The flux flow resistance, for instance, scales with $B \cos \Theta$ when $\Theta$ is varied from $0^{\circ}$ to about $87^{\circ}$. Only in a narrow range below $90^{\circ}$ are deviations observed ${ }^{10}$.

Because the density of pancakes scales as $1 / \cos \Theta$, it seems as if it is possible to ignore the real structure and to consider the pancakes to be stacked on top of each other in the $c$ direction. This also suggests that the positions of the pancakes in each layer are independent of the positions in the adjacent layers. It is as if the layers are totally decoupled and all flux properties are determined for one superconducting layer of thickness equal to the layer spacing
$(1.5 \mathrm{~nm})$. This would account for the fact that large current densities in magnetic fields are only observed at low temperatures where flux creep effects are small.

Referring to the experience with the decoration experiments one may conclude by saying that theoretical models are useful and often essential, but that direct observations are always decisive. Because the Bitter technique is limited to very low fields one may hope that in the future scanning tunnelling microscopes can be developed to such an extent that vortices can be studied in situ at fields of several tesla ${ }^{11}$.

$P . \quad H$. Kes is in the Kamerlingh Onnes Laboratorium, Leiden University, PO Box 9506, 2300 RA Leiden, The Netherlands.

\title{
The question of polarization
}

\section{Graham R. Martin}

THERE have been many investigations of the ways that birds ${ }^{1}$ and insects ${ }^{2}$ can use the Sun's position as a means of orientation for navigation. To function as an orientation cue the Sun does not however need to be perceived directly, because sky-light contains a pattern of polarization which is related to the Sun's position ${ }^{3}$. That some invertebrates ${ }^{4}$ and birds can detect and employ these patterns seemed to have been well established. Now, however, experiments conducted by Coemans and colleagues of the University of Utrecht, reported in Naturwissenschaften, have thrown work on polarization perception in birds into doubt. It may be that the phenomenon will in fact turn out to have been the product of a rather overzealous acceptance of the idea that apparently outstanding behaviour needs a 'super sensory' explanation.

The principal weakness of studies of polarization sensitivity in birds has been the failure of investigators to find a satisfactory way in which light with different planes of polarization could be discriminated. Such mechanisms have been described in certain fish retinae $^{6}$ and in various invertebrate eyes ${ }^{7,8}$ but not yet in birds. It was in looking for such a mechanism that the Utrecht team found that they could not even show that birds were polarization-sensitive.

The main problem in handling polarized light is that it is easy to translate a differentially polarized stimulus into a source of differential brightness. Light from many sources, such as a slide projector, or light that has passed through interference filters, is partially polarized. So simply putting light from such sources through a polarizing filter can induce subtle brightness patterns which change with the orientation of the filter. If a rotating polarizing filter is used, as was the case in one experimental demonstration of polarization sensitivity in the pigeon Columba livia ${ }^{9}$, then the light pattern can become one that flickers.

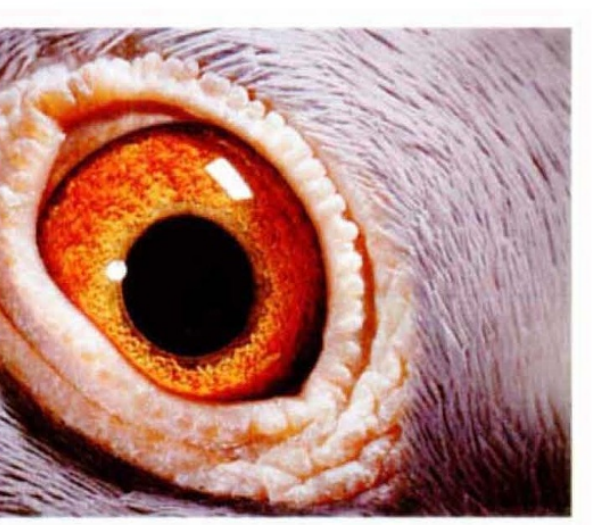

he pigeon - an eye for polarized light?

Another difficulty is that many common surfaces reflect light polarized in different planes unequally. Thus an apparently uniform surface, including a matt black one, uniformly illuminated with polarized light, can become one containing subtle differences in brightness.

Coemans et al. believe that in earlier studies with pigeons, both sources of contamination could have influenced the results. They attempted to overcome these possible artefacts while at the same time reproducing the exact procedures that had previously given positive results. Thus, to eliminate the problem of converting a partially polarized light source into one of differential brightness, the stimulus lights were completely depolarized before being repolarized by filters.

Delius et al. ${ }^{10}$ had demonstrated polarization sensitivity in pigeons using a training procedure where pigeons were taught to make key-peck responses depending upon the plane of polarization of light presented above them. In this study the walls of the response chamber were painted matt black. In the replication of the experiment, Coemans et al. used white blotting paper which was changed after each session. This simple difference in procedure has important consequences. In the original study the matt black surface could have produced differential reflection of the polarized light. Furthermore, such a surface may have become more reflective owing to polishing by the birds' feathers if it had not been renewed regularly. Simply changing the white paper lining of the chamber each day greatly reduced the chances that birds could learn to respond to a differential brightness cue caused by reflections within the chamber.

Using these procedures the Utrecht team were unable to find any evidence of polarization sensitivity in pigeons, although the birds did show rapid learning of other simple visual discriminations in the same apparatus. Coemans et al. were also unable to find any evidence that the electroretinogram of the pigeon eye showed any differential response to polarized light as had previously been reported $^{9,10}$, even though they used stimuli with spectral properties identical to those of the original studies.

The new investigations with pigeons also bring into question the results of field experiments with other birds, such as blackcaps (Sylvia atricapilla) and white-throated sparrows (Zonotichia albicollis), which suggest that the polarization pattern of sky-light influences their orientation behaviour ${ }^{11-13}$. These studies have involved making birds, which are in a state of migratory readiness, view a large section of the sky through sheets of polarizing filter. There is no doubt that the orientation behaviour of some, but not all ${ }^{14}$, birds is influenced by the orientation of such filters. But in the light of the findings from Utrecht it would be prudent not to assume that it is influenced simply by the plane of polarization.

Graham R. Martin is in the Schools of Biological Sciences and of Continuing Studies, University of Birmingham, Edgbaston, Birmingham B15 2TT, UK.

1. Wiltschko, W. \& Wiltschko, R. Trends Ecol. Evol. 3, 13-16 (1988).

2. Wehner, R. NeujBI. Naturf. Ges Zürich 184, 1-132 (1982).

Brines, M. \& Gould, J. Science 206, 571-573 (1979).

4. Fent, K. J. comp. Physiol. A158, 145-150 (1986).

5. Coemans, M., Vos, J. \& Nuboer, J. Naturwissenschaften 77, 138-142 (1990).

6. Hawryshyn, C. W. \& McFarland, W. N. J. comp Physiol. 160. 459-465 (1987).

. Waterman, T. H. Handbook of Sensory Physiology Vol. VII/ $68,281-469$ (1981).

8. Fent, K. \& Wehner, R. Science 228, 192-194 (1985).

9. Kreithen, M. \& Keeton, W. J. comp. Physiol. A89, 73-82 (1974).

10. Delius, J., Perchard, R. \& Emmerton, J. J. comp. physiol. Psychol. 90, 560-571 (1976).

11. Moore, F. Biol. Rev. 62, 65-75 (1987)

12. Able, K. P. J. exp. Biol. 141, 241-251 (1989).

13. Heibig, A. J. \& Wiltschko, W. Naturwissenschaften 76, 227-229 (1989).

14. Sandberg, R. Condor 90, 267-271 (1988). 